\title{
Glibenclamide alleviates inflammation in oleic acid model of acute lung injury through NLRP3 inflammasome signaling pathway
}

This article was published in the following Dove Press journal:

Drug Design, Development and Therapy

\author{
Hong Chen ${ }^{1,2, *}$ \\ Yongjie Ding ${ }^{1,2, *}$ \\ Wei Chen ${ }^{1,2}$ \\ Yun Feng ${ }^{1,2}$ \\ Guochao Shi ${ }^{1,2}$ \\ 'Department of Respiration and Critical \\ Care Disease, Ruijin Hospital, School of \\ Medicine, Shanghai Jiaotong University, \\ Shanghai 20025, People's Republic of \\ China; ${ }^{2}$ Institute of Respiratory Diseases, \\ School of Medicine, Shanghai Jiaotong \\ University, Shanghai 20025, People's \\ Republic of China \\ *These authors contributed equally to \\ this work
}

Background: Pulmonary fat embolism (PFE) is one of the important causes of acute lung injury (ALI), but its pathogenesis is unclear. In recent years, it has been found that the NLRP3 inflammasome is closely related to inflammatory response. However, there are no reports about the involvement of NLRP3 in PFE- associated ALI. Glibenclamide is a kind of hypoglycaemic drug with anti-inflammatory effect. It has been reported to have the antiinflammatory effect related to inhibiting NLRP3.

Objective: To determine whether NLRP3 inflammasome was involved in ALI induced by PFE or whether glibenclamide had therapeutic effects on such lung injury, we designed this experiment.

Materials and methods: The rat model of intravenous injection of oleic acid (OA) was used to simulate PFE. Rats were divided into three groups: control, OA and glibenclamide treatment group. Blood free fatty acid (FFA) concentration was determined by ACS-ACOD. Histopathological examinations were taken to assess the severity of lung injury. The expression of NLRP3 pathway and its downstream products were analyzed by IHC, WB, qPCR and ELISA. Results: Four hours after intravenous OA injection, the typical pathological manifestations of ALI accompanied by elevated levels of plasma FFAs were found. The activity of NLRP3 inflammasomes increased in OA group, too. Pretreatment with glibenclamide partly inhibited the increase in NLRP3, caspase-1 and IL- $1 \beta$ expression induced by OA, simultaneously attenuated the lung injury. But it has little effect on the expression of Toll-like receptor 4 (TLR4) expression in this experiment.

Conclusion: NLRP3 inflammasome, one of the main components of innate immune response, involved in ALI induced by OA. Glibenclamide can alleviate this kind of ALI by inhibiting rather the NLRP3/caspase-1/IL-1 $\beta$ signaling pathway than the levels of FFAs or TLR4 pathway.

Keywords: glibenclamide, oleic acid, acute lung injury, oxidative stress, NLRP3 inflammasome

\section{Introduction}

Pulmonary fat embolism (PFE), which often occurs after major trauma-associated long-bone fractures, can cause lethal acute lung injury (ALI) in severe cases. It also occurs in nontraumatic conditions, such as infection, burns and neoplasm. ${ }^{1,2}$ So far, the pathogenesis is not completely clear. There is also a lack of specific therapeutic drugs.

As a classical model, the oleic acid (OA) model was developed to reproduce ALI due to fat embolism. ${ }^{3}$ OA can directly injure the lung cells and triggers activation of different innate immune receptors, which not only induces neutrophil
Correspondence: Yun Feng: Guochao Shi Department of Respiration and Critical Care Disease, Ruijin Hospital, School of Medicine, Shanghai Jiaotong University, Shanghai 20025, People's Republic of China

Email fy01057@I63.com; shiguochao@hotmail.com 
accumulation but increases inflammatory mediator production, including TNF- $\alpha$, IL- 6 and IL- $1 \beta .{ }^{4}$

The nucleotide-binding oligomerization domain-like receptors (NLRs) are germline-encoded pattern recognition receptors (PRRs) that take part in innate immune responses. The main function of NLRs is to modulate the expression of proinflammatory cytokines, such as IL-1 $\beta$ and IL-18. ${ }^{5}$ NLRP3, the most studied NLRs, can be activated by pathogen-associated molecular patterns (PAMPs) during infection and by endogenous damage-associated molecular patterns (DAMPs) during cellular or tissue injury. ${ }^{6}$ Furthermore, it has been proposed that several cellular signals are responsible for NLRP3 activation, including potassium $\left(\mathrm{K}^{+}\right)$efflux, ROS, pore formation in cell membranes, lysosome and mitochondria damage. ${ }^{7-9}$ Glibenclamide, a type of sulfonylurea agent, is the NLRP3 inflammasome blocker, which mainly inhibits NLRP3 activation through the $\mathrm{K}^{+}$channel $\mathrm{P} 2 \mathrm{X} 7 .^{10}$ It has been reported that glibenclamide can partly alleviate paraquatinduced ALI by inhibiting NLRP3, ${ }^{11}$ but its role in PFEinduced ALI has not been reported so far.

We propose a hypothesis that OA can induce inflammation by activating NLRP3 inflammasome, that was tested by using glibenclamide in OA-induced ALI rat model in this study.

\section{Materials and methods}

\section{Animals}

Adult male Sprague-Dawley rats weighing 200-250 $\mathrm{g}$ were purchased from the Shanghai SLAC Laboratory Animal Co., Ltd. (Shanghai, People'sRepublic of China). Rats were housed with free access to food and water, and with a temperature and humidity-controlled environment $\left(20-25^{\circ} \mathrm{C}, 40-70 \%\right.$ humidity). All experimental procedures were approved by and conducted in accordance with the ethics committee of the animal laboratory of Ruijin hospital, Shanghai Jiao Tong University School Medicine (Shanghai, People'sRepublic of China).

\section{Experimental design}

A total of 18 rats were divided into three groups $(n=6 /$ group): control group (NC), OA group and glibenclamide $+\mathrm{OA}(\mathrm{GL}+\mathrm{OA})$ group. All reagents were available for current use. Glibenclamide (J\&K, People'sRepublic of China) was made into a solution of $10 \mathrm{~g} / \mathrm{L}$ concentration according to the methods previously cited. ${ }^{11}$ Rats in the OA group were injected with pure OA (Aladdin,
People'sRepublic of China) slowly through the tail vein at a dose of $0.1 \mathrm{~mL} / \mathrm{kg}$ to produce ALI model, and saline $(7.5 \mathrm{~mL} / \mathrm{kg}$ ) was administered intraperitoneally $1 \mathrm{hr}$ before OA injection. ${ }^{12}$ In the GL+OA group, animals received glibenclamide at a dose of $75 \mathrm{mg} / \mathrm{kg}$ intraperitoneally $1 \mathrm{hr}$ before $\mathrm{OA}(0.1 \mathrm{~mL} / \mathrm{kg})$ injection. In $\mathrm{NC}$ group, saline was used at a dose of $7.5 \mathrm{~mL} / \mathrm{kg}$ intraperitoneally and then $0.1 \mathrm{~mL} / \mathrm{kg}$ intravenously $1 \mathrm{hr}$ later. Four hours later, the rats were anesthetized by pentobarbital sodium $(40 \mathrm{mg} / \mathrm{kg}$ ) and the plasma was collected for evaluation of non-esterified fatty acid (NEFA) concentration. The middle lobe of the right lung was excised for lung injury evaluation and histopathology. The left lung was excised and processed into homogenate for further testing. ${ }^{13}$

\section{Plasma NEFA quantification}

Plasma concentration of NEFA was determined by the ACS-ACOD method: NEFA in the sample was reacted by acyl-CoA synthase (ACS) to form acyl-CoA, which is oxidized by acyl-CoA oxidase (ACOD) to form hydrogen peroxide. Under the action of peroxidase, the resulting hydrogen peroxide reacts with 3-methyl-N-ethyl-N (betahydroxyethyl methyl)-aniline and 4-aminoantipyrine to form cyan violet pigment. The NEFA concentration in the sample was calculated by measuring the absorbance value of this purple pigment, according to the manufacturer's protocol (BioTNT, People's Republic of China). The detection wavelength was $550 \mathrm{~nm}$. The unit of concentration was $\mathrm{mEq} / \mathrm{L}$.

\section{Histopathological examination}

The middle lobe of the right lung tissue samples was fixed in $10 \%$ Faure Marin solution at $4^{\circ} \mathrm{C}$, then processed for paraffin embedding. The paraffin-embedded tissue blocks were sliced at a thickness for $4 \mu \mathrm{m}$ for histopathological examination. Routine H\&E staining was performed for lung injury evaluation by the pathologists who were blinded for group identity. The histology scoring system was used to evaluate lung injury. ${ }^{11,14}$ Four pathological parameters were scored: 1) alveolar congestion, 2) hemorrhage, 3) leukocyte infiltration and 4) thickness of alveolar wall/hyaline membrane formation. Each category was graded using a 4-point scale: $0=$ minimal damage, $1=$ mild damage, $2=$ moderate damage and $3=$ maximal damage. The total histology score was expressed as the sum of the score for all parameters. Three slides of each rat were prepared for evaluation. 
Immunohistochemistry (IHC) was performed to determine the protein expression of caspase-1, NLRP3 and TLR4. The paraffin sections were pretreated at $62^{\circ} \mathrm{C}$ for 30 mins, then dewaxed in xylene, hydrated and washed. Hydrogen peroxide solution was used to inhibit the endogenous peroxidase. The sections were incubated with specific primary antibodies overnight at $4^{\circ} \mathrm{C}$ : anti-caspase- 1 antibody (Abcam, Cambridge, UK) (1:200), anti-NLRP3/NALP3 antibody (Novus Biologicals, USA) (1:100) and anti-TLR4 antibody (Novus Biologicals, USA) (1:200). Then, membranes were washed thoroughly with phosphate-buffered saline solution. The secondary antibodies (K500711 kit, Dako, Denmark) were added and incubated at $37^{\circ} \mathrm{C}$ for 30 mins. Diaminobenzidine was added and the sections were counterstained with hematoxylin to visualize the reaction products. All the sections were semiquantitatively analyzed by Image-Pro Plus version 6.0 software. The integrated IOD/area (density mean) was measured by evaluating the staining in images at $\times 200$ magnification. Three slides of each rat were prepared for evaluation.

\section{Malondialdehyde (MDA) levels and MPO activity assays}

MDA levels of the rat left lung homogenate were determined by the thiobarbituric acid method. The detection wavelength was $535 \mathrm{~nm}$. Myeloperoxidase (MPO) activity was detected by spectrophotometric method and the detection wavelength was $450 \mathrm{~nm}$. Both procedures were performed according to the manufacturer's protocol (BioTNT, People'sRepublic of China). ${ }^{11}$ The units of concentration were $\mathrm{nmol} / \mathrm{mgprot}$ for $\mathrm{MDA}$ and $\mathrm{U} / \mathrm{g}$ (net weight) for MPO.

\section{NLRP3 and caspase-I mRNA expression analyses}

Trizol method was used to extract RNA from the left lung tissue homogenate (modification of Chomczynski $\mathrm{P}$ and Sacchi N. 1987). The concentration of RNA and the value of A260/280 were measured by NanoDrop DN-1000 (USA). The reverse transcription system was conducted as follows: $2 \mu \mathrm{g}$ RNA was added to $1 \mu \mathrm{L}$ Oligo dT, then DEPC- $\mathrm{ddH}_{2} \mathrm{O}$ was added to $13 \mu \mathrm{L}$, subjected to $65^{\circ} \mathrm{C}$ for 10 mins, then quickly cooled for 2 mins on ice. In the aforementioned system, $4 \mu \mathrm{L}$ 5X M-MLV Buffer, 2 Ll PCR Nucleotide Mix, 0.5 Ll RNase Inhibitor and $0.5 \mu \mathrm{L} \mathrm{M}-\mathrm{MLV}$ were added and incubated at $55^{\circ} \mathrm{C}$ for $30 \mathrm{mins}$, then $85^{\circ} \mathrm{C}$ for 10 mins. Finally, the cDNA template was obtained using the Transcriptor First Strand cDNA Synthesis Kit (Roche, Switzerland). A two-step qPCR reaction was carried out as follows (reaction system of $20 \mu \mathrm{L}$ ): cDNA template $1.0 \mu \mathrm{L}$, SYBR Premix Ex Taq (2X) $10.0 \mu \mathrm{L}$, PCR forward primer $(2 \mu \mathrm{M}) 1 \mu \mathrm{L}$, PCR reverse primer $(2 \mu \mathrm{M})$ $1 \mu \mathrm{L}$ and DEPC-ddH $\mathrm{H}_{2} \mathrm{O} 7 \mu \mathrm{L}$. Fluorescence quantitative PCR (ABI 7500, USA) was used to amplify cDNA for 40 cycles $\left(95^{\circ} \mathrm{C}\right.$ for $10 \mathrm{mins}, 95^{\circ} \mathrm{C}$ for $15 \mathrm{~s}, 60^{\circ} \mathrm{C}$ for $\left.1 \mathrm{mins}\right)$. The data were analyzed with the relative quantitative analysis $\left(2^{-\Delta \Delta \mathrm{Ct}}\right)$ method, and GAPDH was used as the reference housekeeping gene. The following primers were used: NLRP3 forward primer, 5'-CCTCAACAGACGCTACACCC-3' and reverse primer 5'-TCCTGCCAATGGTCAAGAG-3' (size, 89 bp); caspase-1 forward primer, 5'-CGGGCAAGCCAGATGTTTA-3' and reverse primer, 5'-CGCCACCTTCTTTGTTCAGTT-3' (size, 135 bp); GAPDH forward primer, 5'-AA GGTCGGTGTGAACGGATTTG-3' and reverse primer, 5' TGTAGTTGAGGTCAATGAAGGGGTC-3’ (size, 115 bp).

\section{Western blot (WB)}

Expressions of NLRP3, caspase-1 and TLR-4 levels were studied by WB analysis. Protein was extracted from total tissues and washed in PBS, suspended in lysis buffer containing $50 \mathrm{mM}$ Tris- $\mathrm{HCl}$ (pH 7.5), 1\% NP-40, $2 \mathrm{mM}$ EDTA, $10 \mathrm{mM} \mathrm{NaCl}, 10 \mu \mathrm{g} / \mathrm{mL}$ aprotinin, $10 \mu \mathrm{g} / \mathrm{mL}$ leupeptin, $1 \mathrm{mM}$ DTT, $0.1 \%$ SDS and $1 \mathrm{mM}$ PMSF, and placed on ice for 30 mins. After centrifugation for 15 mins at $4^{\circ} \mathrm{C}$, the supernatant was collected. Protein concentrations were determined using a BCA assay (Pierce, Rockford, IL, USA). For WB analysis, equal amounts of proteins $(50 \mu \mathrm{g})$ were separated by SDS-polyacrylamide gel electrophoresis and transferred to aThe membrane was blocked using blocking buffer (5\% non-fat dry milk and $1 \%$ Tween-20 in PBS) for $1 \mathrm{hr}$ at room temperature, then incubated with appropriate primary antibodies: anti-NLRP 3 (Novus Biologicals, USA) (1:500), anti-caspase-1 (CST, USA) (1:1,000) and anti-TLR4 (Novus Biologicals, USA) $(1: 1,000)$ in blocking buffer overnight at $4^{\circ} \mathrm{C}$. The blot was then incubated with appropriate alkaline phosphatase (AP)-conjugated secondary antibody: anti-rabbit $\operatorname{IgG}$, HRP-linked antibody (CST, USA) $(1: 2,000)$ and detected in $5 \mathrm{~mL}$ AP buffer containing $16.5 \mu \mathrm{L}$ BCIP and $33 \mu \mathrm{L}$ NBT at room temperature for 10-20 mins, then photographed. GAPDH (KC, People'sRepublic of China) $(1: 5,000)$ was used as a loading control.

\section{IL-I $\beta$ and IL-I 8 ELISA assays}

IL-1 $\beta$ and IL-18 concentrations in the left lung tissue homogenate were analyzed by using the double antibody sandwich method in ELISA (BioTNT, People'sRepublic of China), 
according to the manufacturer's protocol. ${ }^{11}$ The OD value of the well was measured at a wavelength of $450 \mathrm{~nm}$ on the ELISA tester. The concentration of the diluted samples (X value) was obtained according to the standard curve of OD. The final sample concentration was $\mathrm{X}$ value * the multiplier of dilution.

\section{Statistical analyses}

Statistical analyses were carried out using SPSS 22.0. The data were expressed as median (range, min-max). Kruskal-Wallis and median test (k samples) followed by multiple comparison tests were used to compare the results obtained in $\mathrm{NC}, \mathrm{OA}$ and GL+OA groups. $P<0.05$ was considered to indicate a statistically significant difference.

\section{Results}

Glibenclamide does not affect the level of FFAs induced by OA

We measured NEFA level in rat plasma to reflect FFAs level. As expected, the level increased significantly $(P=0.022)$ after $\mathrm{OA}$ intravenous injection. Even after 4 hrs, we could still detect higher FFAs level compared with the control group (Figure 1A). The glibenclamide did not attenuate the FFAs level compared with OA group $(P=1.000)$, and the level of FFAs was higher than that in NC group $(P=0.028)$ (Figure 1A).

\section{Glibenclamide alleviates $A L I$ induced by OA}

In NC group, the lung tissue structure was normal (Figure 1B). But the early typical pathological changes of ALI were visible in OA group (Figure 1C): pulmonary interstitial edema and the formation of hyaline membrane; infiltration of more neutrophils and mononuclear cells in the pulmonary interstitial and alveolar cavities; vasculitis and vascular extravasation. The lung injury score in OA group was much higher than in NC group $(P=0.000)$ (Figure 1E). Pretreatment with glibenclamide significantly alleviated the lung injury (Figure 1D), as same as the lung injury score $(P=0.017)$ shown in Figure $1 \mathrm{E}$, though the lung injury was still severe (Figure 1D) and the lung injury score was higher $(P=0.000)$ (Figure $1 \mathrm{E})$ compared with $\mathrm{NC}$ group. It implied that activation of NLRP3 inflammasome involved in this type of endogenous inflammatory response and glibenclamide administration partly reversed the OAinduced lung injury. Combining the difference of NEFA level in three groups, we hypothesized that glibenclamide attenuated OA-induced lung injury by rather inhibiting downstream inflammatory reaction than reducing FFAs level.

\section{Glibenclamide inhibits the oxidative stress response induced by $\mathrm{OA}$}

MDA is a product of ROS lipid peroxidation, which indirectly reflects the degree of tissue peroxidation. MPO reacts to the degree of neutrophil activity. Neutrophils are the main inflammatory cells that produce ROS. The concentration of MDA and the activity of MPO in the rat lung tissue of OA group were significantly higher compared with those in NC group (Figure 2). These indicated oxidative stress activity increased in OA group. However, in GL+OA group, MDA and MPO decreased in varying degrees, suggesting that glibenclamide could partially inhibit oxidative stress response (Figure 2).

\section{Glibenclamide decreases the protein and mRNA expression levels of NLRP3 and caspase-I induced by OA}

NLRP3 and caspase-1 are the main components of NLRP3 inflammasome complex. Compared with the control group, NLRP3 and caspase-1 protein and mRNA levels significantly
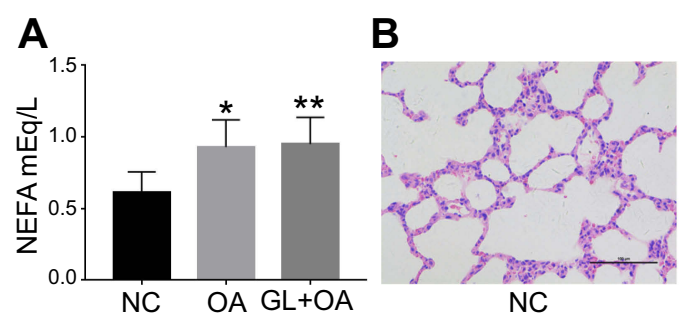

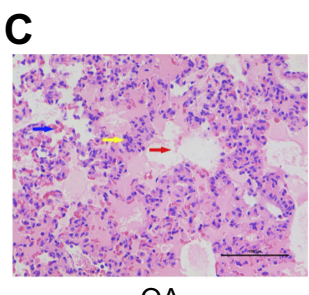

OA

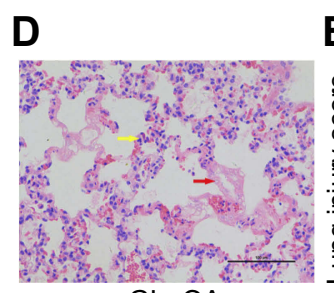

$\mathrm{GL}+\mathrm{OA}$

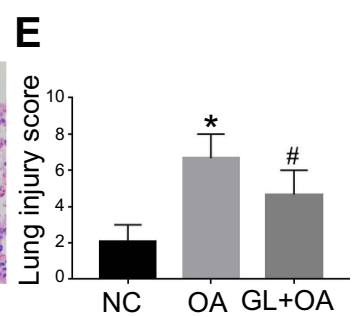

Figure I NEFA level and lung injury manifestations in three groups. (A) Plasma level of NEFA in three groups. (B-D) Histopathological changes of lung tissues in three groups (HE, X200). (B) The control group shows normal lung architecture. (C) The OA group shows the early pathological changes of ALI. (D) The GL+OA group shows less damage than OA group. Blue arrows denote bleeding; yellow arrows point to inflammatory cells; red arrows indicate hyaline membrane. (E) Lung injury scores in three groups. Data are presented as median (range, min-max) ( $\mathrm{n}=6$ /group). ${ }^{*} P=0.022(\mathrm{~A}),{ }^{*} P=0.000(\mathrm{E})$, $\mathrm{OA}$ group versus $\mathrm{NC}$ group. ** $P=0.028(\mathrm{~A}), \mathrm{GL}+\mathrm{OA}$ group versus $\mathrm{OA}$ group. ${ }^{\#}=0.017(\mathrm{E}), \mathrm{GL}+\mathrm{OA}$ group versus $\mathrm{NC}$ group.

Abbreviations: NC, negative control; OA, oleic acid; GL, glibenclamide; NEFA, non-esterified fatty acid. 
A

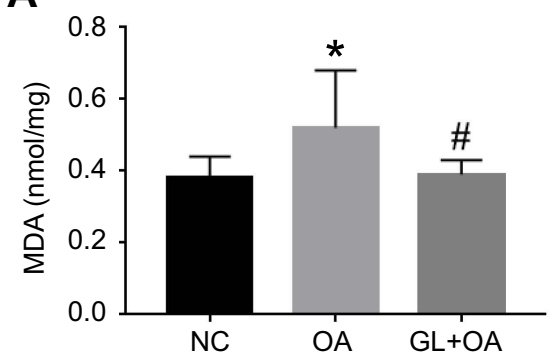

B

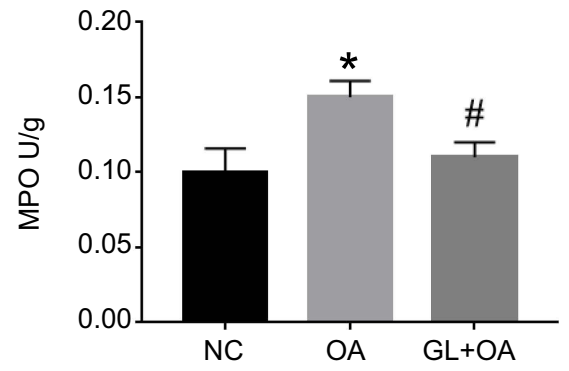

Figure 2 MDA levels and MPO activity in three groups. OA increased the MDA level (A) and MPO activity (B), but the glibenclamide inhibited both of them. Data are presented as the median (range, min-max) ( $n=6$ /group). ${ }^{*} P=0.028$ (A), ${ }^{*} P=0.002(\mathbf{B})$, OA group versus $\mathrm{NC}$ group. ${ }^{\#} P=0.028(\mathbf{A}),{ }^{\#} P=0.045$ (B), $G L+O A$ group versus OA group.

Abbreviations: MDA, malondialdehyde; MPO, myeloperoxidase; NC, negative control; OA, oleic acid; GL, glibenclamide.

increased in OA group, evaluated by IHC (Figure 3A-H), PCR (Figure 4A and B) and WB (Figure 4C and D). But the glibenclamide, as a kind of inflammasome blocker, inhibited these expressions in GL+OA group (Figures 3 and 4). TLR-4 protein expression increased slightly in OA group, but glibenclamide could not inhibit its expression (Figure 3I-L, Figure

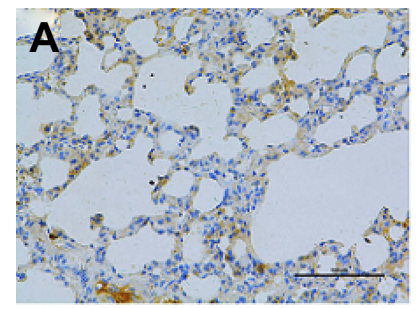

NC

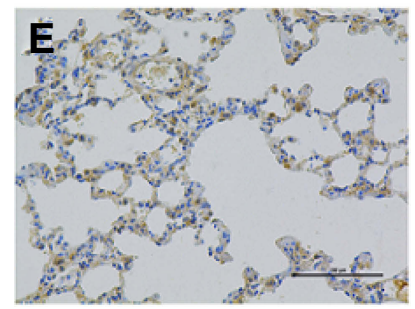

NC

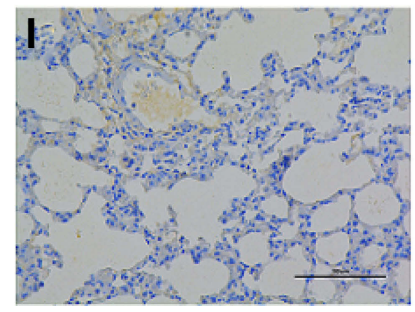

NC

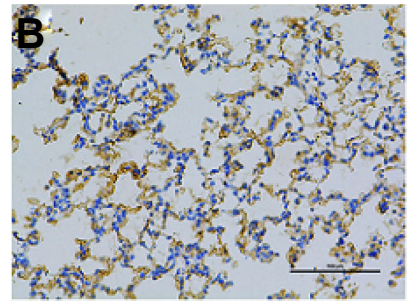

$\mathrm{OA}$

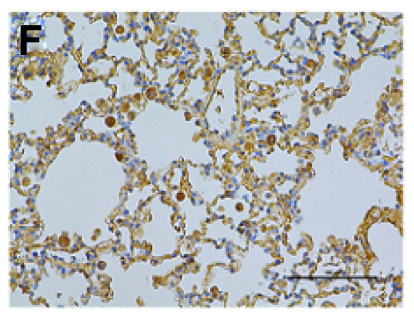

$\mathrm{OA}$

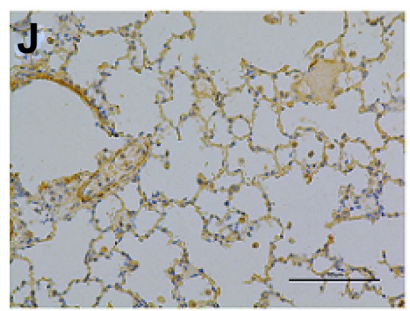

$\mathrm{OA}$
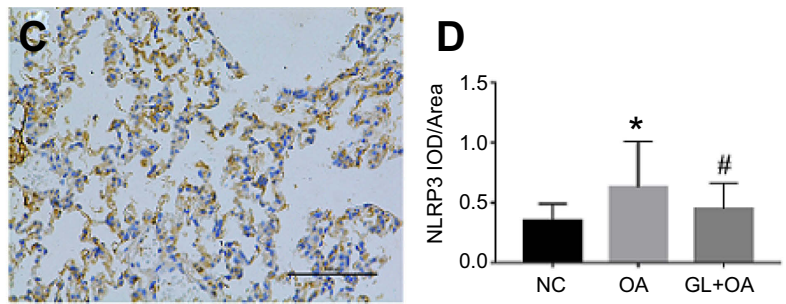

$\mathrm{GL}+\mathrm{OA}$
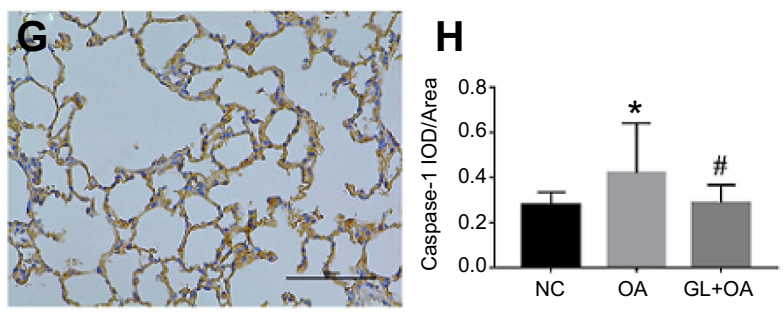

$\mathrm{GL}+\mathrm{OA}$
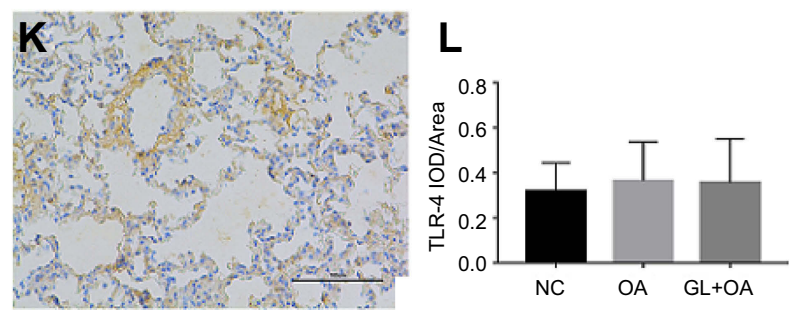

$\mathrm{GL}+\mathrm{OA}$

Figure 3 NLRP3 and caspase-I expression in three groups by IHC. (A, E and I) control group; (B, F and J) OA group; (C, G and K) GL+OA group. (A-D) NLRP3 and $(\mathbf{E}-\mathbf{H})$ caspase-I staining intensities were strongest in $\mathrm{OA}$ group, attenuated in $\mathrm{GL}+\mathrm{OA}$ group and weakest in control group. $* P=0.000(\mathbf{D}), * P=0.000(\mathbf{H}), \mathrm{OA}$ group versus NC group. ${ }^{\#} P=0.013$ (D), ${ }^{\#} P=0.000$ (H), GL+OA group versus OA group. (I-L) TLR4 staining intensity was not changed obviously in three groups $(P=0.277)$.

Abbreviations: NC, negative control; OA, oleic acid; GL, glibenclamide, IHC, immunohistochemical analyses. 

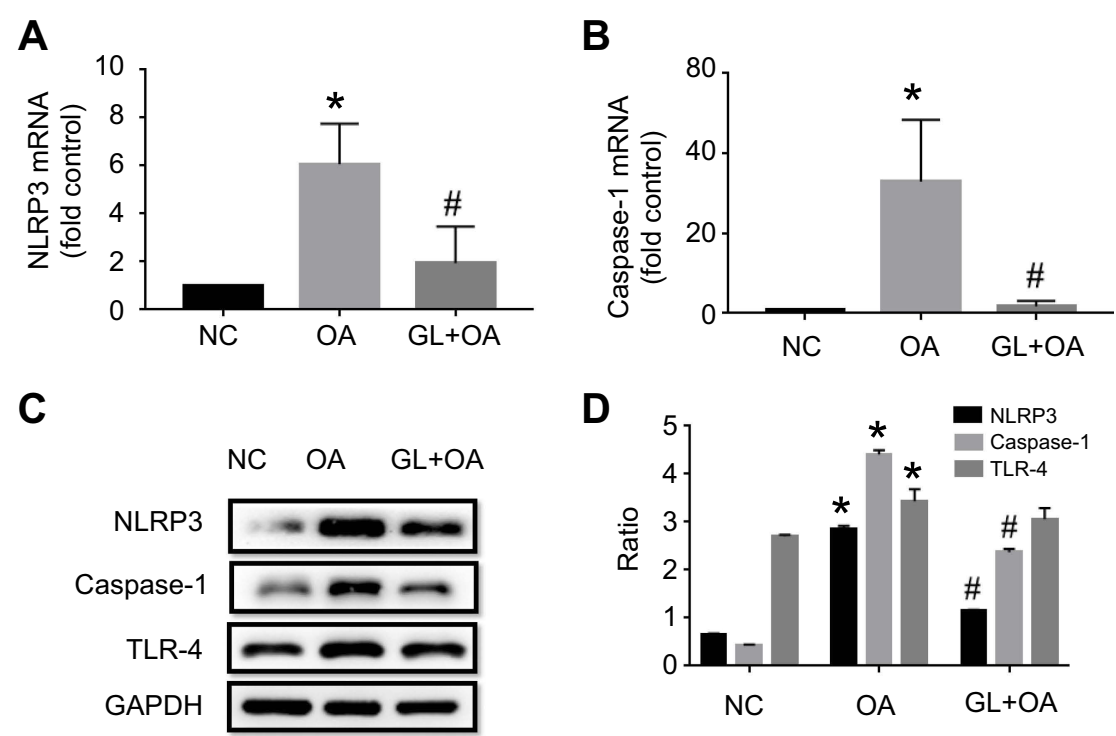

Figure 4 NLRP3, caspase-I and TLR4 mRNA and protein expression in three groups. NLRP3, caspase-I and TLR4 expression levels were assessed by qPCR (A and B) and WB (C and $\mathbf{D})$ in three groups. The levels of mRNA expression (A and $\mathbf{B})$ and protein $(\mathbf{C}$ and $\mathbf{D})$ of NLRP3 and caspase-I increased in OA group, but were inhibited by glibenclamide. The protein level of TLR4 increased slightly in OA group, and glibenclamide could not inhibit it by $W B(\mathbf{C}$ and $\mathbf{D})$. $* P=0.002(\mathbf{A}), * P=0.003(\mathbf{B})$, $* P<0.05(\mathbf{D}), \mathrm{OA}$ group versus $\mathrm{NC}$ group. ${ }^{\#} P=0.002$ (A), ${ }^{\#} P=0.025$ (B), ${ }^{\#} P<0.05$ (D), GL+OA group versus $O A$ group.

Abbreviations: NC, negative control; OA, oleic acid; GL, glibenclamide; qPCR, quantitative-polymerase chain reaction; WB, Western blot analysis.

4C and D). We speculated that rather NLRP3 inflammasome which could be inhibited by the glibenclamide than TLR4 receptors, involved in OA-induced ALI.

\section{Glibenclamide decreases IL-I $\beta$ expression induced by $\mathrm{OA}$}

We tested the IL-1 $\beta$ and IL-18 expression of the lung tissue in three groups. As a downstream product of NLRP3 inflammasome activation pathway, IL-1 $\beta$ increased significantly in the OA group. Glibenclamide inhibited the expression of this proinflammatory cytokine (Figure 5A). However, no significant change was observed in IL-18 in OA group compared with NC group (Figure 5B). These suggested that at the early stage of OA-induced ALI, NLRP3 inflammasome pathway increased IL-1 $\beta$ expression more significantly than IL-18, and glibenclamide could inhibit the increase of IL-1 $\beta$ rather than IL-18 through NLRP3 pathway, thus alleviating OA-induced ALI.

\section{Discussion}

We successfully created rat ALI model by OA intravenous injection, characterized by alveolar and interstitial edema, alveolar collapse, inflammatory cell infiltration, intravascular congestion and alveolar hemorrhage accompanied by the increase of FFAs level. In another animal model of fat embolism, FFAs increased after $4 \mathrm{hrs}$, reached a peak at 24 hrs, and consisted with the decrease of arterial oxygen partial pressure and clinical manifestations, ${ }^{15}$ that was same as our results. Furthermore, it is reported that
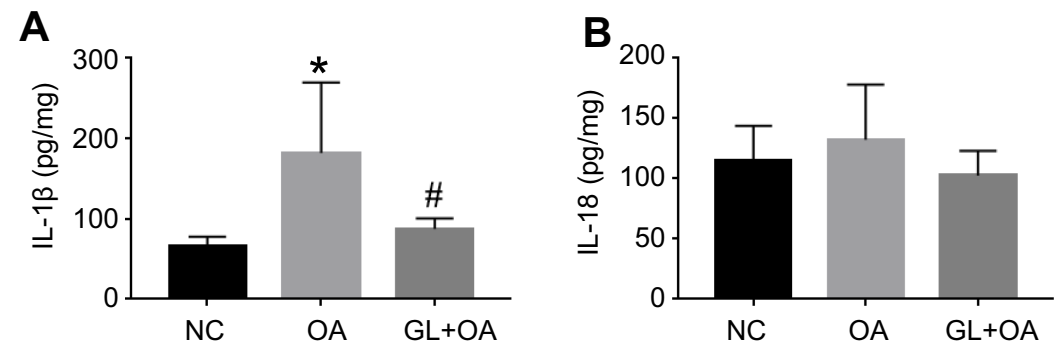

Figure $5 \mathrm{IL}-\mathrm{I} \boldsymbol{\beta}$ and IL- 18 expression of lung tissue homogenate in three groups. (A) IL-I $\boldsymbol{\beta}$ was increased in the OA group compared with the control group $(* P=0.002)$, and attenuated in $\mathrm{GL}+\mathrm{OA}$ group compared with the OA group ( $\left.{ }^{\#} P=0.017\right)$, as evaluated by ELISA. (B) There was no difference in IL- 18 expression in the three groups $(P=0.119)$. Abbreviations: NC, negative control; OA, oleic acid; GL, glibenclamide. 
increasing plasma FFAs are closely related to the severity of disease in sepsis, leptospirosis, pancreatitis and other critically ill diseases. ${ }^{16-18}$

OA, the main component of FFAs, combines with albumin without toxicity under physiological conditions. ${ }^{19}$ However, it can cause ALI when a large amount of unbound OA gathers in lung tissue under pathological conditions. ${ }^{20}$ In PFE, FFAs originate from local hydrolysis of a triglyceride emboli by intrapulmonary lipase, together with the excessive mobilization of FFAs from the peripheral adipose tissue by stress hormones. $^{21}$

FFAs activate the oxidative stress reaction in OAinduced $\mathrm{ALI},{ }^{22-24}$ which we also observed in this study by measuring MDA and MPO. Through mitochondrial electron leakage, cell plasma membrane NADPH oxidase and endoplasmic reticulum stress (ER stress), FFAs generate a large amount of $\operatorname{ROS}^{25-27}$ that can directly oxidize and damage DNA, proteins and lipids which act as functional molecular signals to activate a variety of signaling pathways in cells, ${ }^{4,28,29}$ such as PRR.

The NLRP3 inflammasome, as the cytosolic PRR, can recognize PAMP or DAMP and then activate downstream caspase-1. ROS and FFAs-associated oxidative stress productions, such as mitochondrial DNA are the main activators of NLRP3 inflammasome. ${ }^{8,30-32}$ In this study, after OA injection NLRP3 inflammasome activation increased significantly and was accompanied by the activation of caspase- 1 processing that could cleave inactive IL- $1 \beta$ precursor into its bioactive form. ${ }^{33,34} \mathrm{IL}-1 \beta$ is also known as an indicator of NLRP3 inflammasome induction. ${ }^{35}$ IL-18, one of the other NLRP3 downstream proinflammatory cytokines, is essential for some ALI models. ${ }^{11,36-39}$ But no obvious changes of IL-18 levels were found in this study.

TLR4 is an another PRRs located on the cell membrane, which is involved in innate immunity and plays an important role in different types of lung injury. ${ }^{37,38}$ However, no association with TLR4 was found in OA-induced lung injury model in vivo. ${ }^{4}$ In our study, we did not find prominent increase of TLR4 expression in OA group, either. FFAs were once thought to produce inflammation by activating TLRs. This hypothesis originated from the observation that saturated fatty acid was necessary for lipopolysaccharide -induced inflammation. ${ }^{40}$ It is now clear that palmitic acid (PA) is not an agonist of TLR4, nor can it activate TLR signals. ${ }^{41}$ However, TLR activation is still crucial for FFAs-induced inflammation, because it can lead to metabolic reprogramming and promotes PAinduced inflammation. ${ }^{41}$ This may also explain why the level of IL-18 is not elevated obviously in this study, because the activation of IL-18 but not IL-1 $\beta$ depends on TLR4 pathway. ${ }^{42}$ It is speculated that OA could transfer the extracellular signals into cell through the cell membrane $\mathrm{Na} / \mathrm{K}$-ATPase and free fatty acid receptor 1 , then activate the downstream MAPK-ERK1/2-NF- $\kappa$ B pathway, which eventually leads to a series of inflammatory reactions. ${ }^{24}$ The NF- $\kappa$ B signal pathway is essential for NLRP3 activation in the "priming" process (the first step). ${ }^{34}$ D'Espessailles A also confirmed that ERK1/2 was involved in the activation of NLRP3 in adipose cells. ${ }^{43}$ We hypothesized that ERK1/2 could mediate in the activation of NLRP3 in OA-induced lung injury, too.

Glibenclamide preconditioning inhibited the activity of NLRP3 inflammasome, thereby partly alleviated the manifestations of OA-induced ALI consistent with previous observations in other types of ALI models. ${ }^{11}$ A great deal of evidence shows that glibenclamide has anti-inflammatory effect not only in respiratory, but also in digestive, urinary, heart and central nervous system diseases and ischemiareperfusion injury. ${ }^{44-48}$ The mechanism of glibenclamide inhibiting NLRP3 activity is still unclear. It is speculated that it may block potassium ATP channel, block $\mathrm{K}^{+}$outflow, change membrane potential and inhibit $\mathrm{Ca}^{2+}$ influx mediated by $\mathrm{P} 2 \mathrm{X} 7$ receptor, thus inhibiting NLRP3 inflammasome activity. ${ }^{49}$ In addition, glibenclamide has been reported to inhibit oxidative stress by increasing the activities of antioxidant enzymes, such as glutathione peroxidase, superoxide dismutase and catalase yet. ${ }^{50,51}$ The oxidative stress product is the strong activator of NLRP3 activation. In this study, it was also observed that glibenclamide slightly attenuated ROS response. In short, we found that glibenclamide partially alleviated the ALI caused by OA, which may provide novel strategies for the treatment of PFE in the future.

\section{Conclusion}

we demonstrated that the NLRP3/caspase-1 pathway was involved in the development of the OA/ROS/IL-1 $\beta$ inflammatory reaction in OA-induced ALI model. The NLRP3 inhibitor glibenclamide can alleviate this kind of ALI. Combined with Goncalves-de-Albuquerque 's study, ${ }^{20}$ we proposed the mechanism of NLRP3 inflammasome in OA induced ALI in Figure 6.

\section{Limitation}

In this experiment, we initially observed the role of NLRP3 inflammasome in OA- induced ALI. There were some limitations in this study. First, we did not carry out 


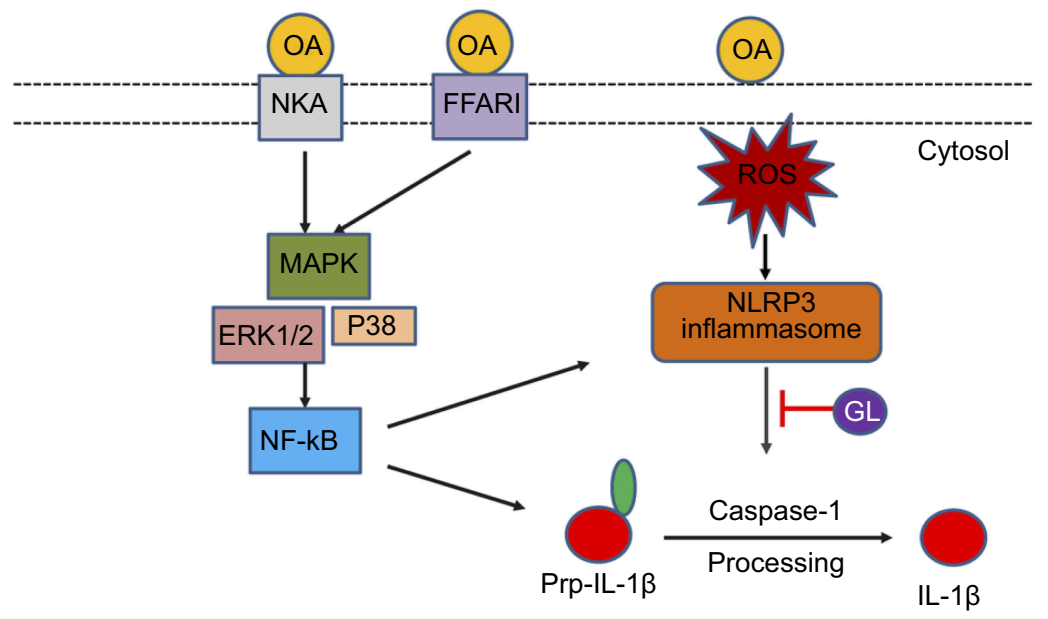

Figure 6 Model of NLRP3 inflammasome activation involved in OA-induced ALI rat model. On the one hand, OA activates the MAPK-ERKI/2-NF-KB pathway through cell membrane NKA and FFARI, produces a large number of pro-IL-I $\beta$ and may increase the formation and assembly of NLRP3 inflammasome complex. On the other hand, OA activates NLRP3-caspase-I pathway through ROS, and eventually produces a large number of bioactive IL-I $\beta$, leading to ALI.

Abbreviations: OA, oleic acid; NKA, $\mathrm{Na}^{+} / \mathrm{K}^{+}$-ATPase; MAPK, mitogen-activated protein kinases; FFARI, free fatty acid receptor I; ERKI/2, extracellular signal-regulated kinases; NF-KB, nuclear factor kappa-light-chain-enhancer of activated B cells; GL, glibenclamide.

in vitro studies. Second, a small sample size may lead to deviation of results. Third, we did not investigate the effect of glibenclamide after OA administration. In addition, further studies are needed to prove whether MAPKERK1/2 pathway is involved in initiating NLRP3 inflammasome in OA-induced ALI model.

\section{Acknowledgments}

This study is supported by Shanghai key discipline for respiratory diseases (Grant No. 2017ZZ02014)

\section{Disclosure}

The authors report no conflicts of interest in this work.

\section{References}

1. Shier MR, Wilson RF. Fat embolism syndrome: traumatic coagulopathy with respiratory distress. Surg Annu. 1980;12:139-168.

2. Moylan JA, Birnbaum M, Katz A, Everson MA. Fat emboli syndrome. J Trauma. 1976;16:341-347.

3. Schuster DP. ARDS: clinical lessons from the oleic acid model of acute lung injury. Am J Respir Crit Care Med. 1994;149:245-260. doi:10.1164/ajrccm.149.1.8111590

4. Goncalves-de-Albuquerque CF, Silva AR, Burth P, et al. Oleic acid induces lung injury in mice through activation of the ERK pathway. Mediators Inflamm. 2012;2012:1-11. doi:10.1155/2012/ 956509

5. Shaw MH, Reimer T, Kim YG, et al. NOD-like receptors (NLRs): bona fide intracellular microbial sensors. Curr Opin Immunol. 2008;20:377-382. doi:10.1016/j.coi.2008.06.001

6. Lee S, Suh GY, Ryter SW, Choi AMK. Regulation and function of the nucleotide binding domain leucine-rich repeat-containing receptor, pyrin domain-containing-3 inflammasome in lung disease. Am J Respir Cell Mol Biol. 2016;54:151-160. doi:10.1165/rcmb.2015-0231TR
7. Nakahira K, Haspel JA, Rathinam VA, et al. Autophagy proteins regulate innate immune responses by inhibiting the release of mitochondrial DNA mediated by the NALP3 inflammasome. Nat Immunol. 2011;12:222-230. doi:10.1038/ni.1980

8. Zhou R, Tardivel A, Thorens B, Choi I, Tschopp J. Thioredoxininteracting protein links oxidative stress to inflammasome activation. Nat Immunol. 2010;11:136-140. doi:10.1038/ni.1831

9. Chen S, Sun B. Negative regulation of NLRP3 inflammasome signaling. Protein Cell. 2013;4:251-258. doi:10.1007/s13238-013-2128-8

10. Schroder K, Tschopp J. The inflammasomes. Cell. 2010;140:821-832. doi:10.1016/j.cell.2010.01.040

11. Liu Z, Zhao H, Liu W, Li T, Wang Y, Zhao M. NLRP3 inflammasome activation is essential for paraquat-induced acute lung injury. Inflammation. 2015;38:433-444. doi:10.1007/s10753-014-0048-2

12. Huang B, Wang DX, Deng W. Protective effects of dexamethasone on early acute lung injury induced by oleic acid in rats. Int J Clin Exp Med. 2014;7:4698-4709.

13. Zhao Y, Zhang M, Xiong RP, et al. Somatostatin reduces the acute lung injury of mice via increasing the affinity of glucocorticoid receptor. Cell Physiol Biochem. 2016;38:1354-1364. doi:10.1159/ 000443079

14. You Z, Feng D, Xu H, et al. Nuclear factor-kappa B mediates one-lung ventilation-induced acute lung injury in rabbits. $J$ Invest Surg. 2012;25:78-85. doi:10.3109/08941939.2011.603817

15. Yulan Z, Nan L, Qun Z, et al. Determination of free fatty acids in fat embolism syndrome by GC-MS. Chin J Pharm Anal. 1998;S1:44-45.

16. Burth P, Younes-Ibrahim M, Santos MC, et al. Role of nonesterified unsaturated fatty acids in the pathophysiological processes of leptospiral infection. J Infect Dis. 2005;191:51-57. doi:10.1086/426455

17. Goncalves-de-Albuquerque CF, Burth P, Silva AR, Younes-Ibrahim M, Castro-Faria-Neto HC, Castro-Faria MV. Leptospira and inflammation. Mediators Inflamm. 2012;2012:317950. doi:10.1155/2012/317950

18. Nogueira AC, Kawabata V, Biselli P, et al. Changes in plasma free fatty acid levels in septic patients are associated with cardiac damage and reduction in heart rate variability. Shock. 2008;29:342-348. doi:10.1097/shk.0b013e31815abbc6

19. Costanzi S, Neumann S, Gershengorn MC. Seven transmembrane-spanning receptors for free fatty acids as therapeutic targets for diabetes mellitus: pharmacological, phylogenetic, and drug discovery aspects. J Biol Chem. 2008;283:16269-16273. doi:10.1074/ jbc.R800014200 
20. Goncalves-de-Albuquerque CF, Silva AR, Burth P, et al. Acute respiratory distress syndrome: role of oleic acid-triggered lung injury and inflammation. Mediators Inflamm. 2015;2015:260465. doi: $10.1155 / 2015 / 125380$

21. Baker PL, Pazell JA, Peltier LF. Free fatty acids, catecholamines, and arterial hypoxia in patients with fat embolism. $J$ Trauma. 1971;11:1026-1030.

22. Altintas ND, Atilla P, Iskit AB, Topeli A. Long-term simvastatin attenuates lung injury and oxidative stress in murine acute lung injury models induced by oleic acid and endotoxin. Respir Care. 2011;56:1156-1163. doi:10.4187/respcare.00770

23. Liu H, Zhang D, Zhao B, Zhao J. Superoxide anion, the main species of ROS in the development of ARDS induced by oleic acid. Free Radic Res. 2004;38:1281-1287. doi:10.1080/10715760400006940

24. Salman AE, Yetisir F, Kilic M, et al. The impact of pretreatment with bolus dose of enteral glutamine on acute lung injury induced by oleic acid in rats. J Anesth. 2014;28:354-362. doi:10.1007/s00540-013-1745-y

25. Schonfeld P, Wojtczak L. Fatty acids as modulators of the cellular production of reactive oxygen species. Free Radic Biol Med. 2008;45:231-241. doi:10.1016/j.freeradbiomed.2008.04.029

26. Tu BP, Weissman JS. Oxidative protein folding in eukaryotes: mechanisms and consequences. J Cell Biol. 2004;164:341-346. doi: $10.1083 /$ jcb. 200311055

27. Graciano MF, Valle MM, Kowluru A, Curi R, Carpinelli AR. Regulation of insulin secretion and reactive oxygen species production by free fatty acids in pancreatic islets. Islets. 2011;3:213-223.

28. Ballard-Croft C, Wang D, Sumpter LR, Zhou X, Zwischenberger JB. Large-animal models of acute respiratory distress syndrome. Ann Thorac Surg. 2012;93:1331-1339. doi:10.1016/j.athoracsur.2011.06.107

29. Bae H, Jeong CH, Cheng WN, Hong K, Seo HG, Han SG. Oxidative stress-induced inflammatory responses and effects of $\mathrm{N}$-acetylcysteine in bovine mammary alveolar cells. $J$ Dairy Res. 2017;84:418-425. doi:10.1017/S002202991700067X

30. Shimada K, Crother TR, Karlin J, et al. Oxidized mitochondrial DNA activates the NLRP3 inflammasome during apoptosis. Immunity. 2012;36:401-414. doi:10.1016/j.immuni.2012.01.009

31. Horng T. Calcium signaling and mitochondrial destabilization in the triggering of the NLRP3 inflammasome. Trends Immunol. 2014;35:253-261. doi:10.1016/j.it.2014.02.007

32. Iyer SS, He Q, Janczy JR, et al. Mitochondrial cardiolipin is required for Nlrp3 inflammasome activation. Immunity. 2013;39:311-323. doi:10.1016/j.immuni.2013.08.001

33. Latz E, Xiao TS, Stutz A. Activation and regulation of the inflammasomes. Nat Rev Immunol. 2013;13:397-411. doi:10.1038/nri3452

34. Schroder K, Zhou R, Tschopp J. The NLRP3 inflammasome: a sensor for metabolic danger? Science. 2010;327:296-300. doi:10.1126/ science. 1184003

35. Kanneganti TD, Lamkanfi M, Kim YG, et al. Pannexin-1-mediated recognition of bacterial molecules activates the cryopyrin inflammasome independent of toll-like receptor signaling. Immunity. 2007;26:433-443. doi:10.1016/j.immuni.2007.03.008

36. Zhang Q, Wu D, Yang Y, Liu T, Liu H. Dexmedetomidine alleviates hyperoxia-induced acute lung injury via inhibiting NLRP3 inflammasome activation. Cell Physiol Biochem. 2017;42:1907-1919. doi:10.1159/000479609

37. Luo YP, Jiang L, Kang K, et al. Hemin inhibits NLRP3 inflammasome activation in sepsis-induced acute lung injury, involving heme oxygenase-1. Int Immunopharmacol. 2014;20:24-32. doi:10.1016/j. intimp.2014.02.017
38. Xiang M, Shi X, Li Y, et al. Hemorrhagic shock activation of NLRP3 inflammasome in lung endothelial cells. J Immunol. 2011;187:4809-4817. doi:10.4049/jimmunol.1102093

39. Yang X, Sun X, Chen H, et al. The protective effect of dopamine on ventilator-induced lung injury via the inhibition of NLRP3 inflammasome. Int Immunopharmacol. 2017;45:68-73. doi:10.1016/ j.intimp.2017.02.002

40. Wisse BE. The inflammatory syndrome: the role of adipose tissue cytokines in metabolic disorders linked to obesity. $\mathrm{J} \mathrm{Am} \mathrm{Soc}$ Nephrol. 2004;15:2792-2800. doi:10.1097/01.ASN.0000141966. 69934.21

41. Lancaster GI, Langley KG, Berglund NA, et al. Evidence that TLR4 is not a receptor for saturated fatty acids but mediates lipidinduced Inflammation by reprogramming macrophage metabolism. Cell Metab. 2018;27:1096-1110.e1095. doi:10.1016/j.cmet.2018. 03.014

42. Parker JC. Mitochondrial damage pathways in ventilator induced lung injury (VILI): an update. J Lung Health Dis. 2018;2(2):18-22.

43. D‘Espessailles A, Mora YA, Fuentes C, Cifuentes M. Calciumsensing receptor activates the NLRP3 inflammasome in LS14 preadipocytes mediated by ERK1/2 signaling. $J$ Cell Physiol. 2018;233:6232-6240. doi:10.1002/jcp.26490

44. York JM, Castellanos KJ, Cabay RJ, Fantuzzi G. Inhibition of the nucleotide-binding domain, leucine-rich containing family, pyrin-domain containing 3 inflammasome reduces the severity of experimentally induced acute pancreatitis in obese mice. Transl Res. 2014;164(4):259-269. doi:10.1016/j.trsl.2014.06.012

45. Ling MY, Ma ZY, Wang YY, et al. Up-regulated ATP-sensitive potassium channels play a role in increased inflammation and plaque vulnerability in macrophages. Atherosclerosis. 2013;226(2):348-355. doi:10.1016/j.atherosclerosis.2012.11.016

46. Hughes FM Jr, Vivar NP, Kennis JG, et al. Inflammasomes are important mediators of cyclophosphamide-induced bladder inflammation. Am J Physiol Renal Physiol. 2014;306(3):F299F308. doi:10.1152/ajprenal.00297.2013

47. Su WJ, Peng W, Gong H, et al. Antidiabetic drug glyburide modulates depressive-like behavior comorbid with insulin resistance. $J$ Neuroinflammation. 2017;14(1):210. doi:10.1186/s12974-0170985-4

48. Ortega FJ, Gimeno-Bayon J, Espinosa-Parrilla JF, et al. ATPdependent potassium channel blockade strengthens microglial neuroprotection after hypoxia-ischemia in rats. Exp Neurol. 2012;235 (1):282-296. doi:10.1016/j.expneurol.2012.02.010

49. Lamkanfi M, Mueller JL, Vitari AC, et al. Glyburide inhibits the cryopyrin/Nalp3 inflammasome. J Cell Biol. 2009;187(1):61-70. doi:10.1083/jcb. 200903124

50. Abdallah DM, Nassar NN, Abd-El-Salam RM. Glibenclamide ameliorates ischemia-reperfusion injury via modulating oxidative stress and inflammatory mediators in the rat hippocampus. Brain Res. 2011;1385:257-262. doi:10.1016/j.brainres.2011.02.007

51. Nazaroglu NK, Sepici-Dincel A, Altan N. The effects of sulfonylurea glyburide on superoxide dismutase, catalase, and glutathione peroxidase activities in the brain tissue of streptozotocin-induced diabetic rat. J Diabetes Complications. 2009;23(3):209-213. doi:10.1016/j. jdiacomp.2007.09.001 


\section{Publish your work in this journal}

Drug Design, Development and Therapy is an international, peerreviewed open-access journal that spans the spectrum of drug design and development through to clinical applications. Clinical outcomes, patient safety, and programs for the development and effective, safe, and sustained use of medicines are a feature of the journal, which has also been accepted for indexing on PubMed Central. The manuscript management system is completely online and includes a very quick and fair peer-review system, which is all easy to use. Visit http://www. dovepress.com/testimonials.php to read real quotes from published authors. 